\title{
ESTUDIO DEL COMPORTAMIENTO DE DIFERENTES DENSIDADES DE SIEMBRA DE DEDINIOS PARA EL ENGORDE DE TRUCHA ARCO IRIS
}

\author{
STUDY OF THE BEHAVIOR OF DIFFERENT SOWING DENSITIES \\ OF DEADS FOR THE RAINBOW TROUT FAT
}

\author{
Edwin Hitler Muyulema Erazo \\ Marcelo Eduardo Moscoso Gómez \\ Bertha Hiparía Chuva Buele \\ Richar Neboth Muyulema Erazo \\ rimuer1@gmail.com
}


Para citar este artículo:

Muyulema Erazo, E. H., Moscoso Gómez, M. E., Chuva Buele, B. H., \& Muyulema Erazo, R. N. (2021). Estudio del comportamiento de diferentes densidades de siembra de dedinios para el engorde de trucha arco iris. ESPACIO I+D, INNOVACIÓN MÁS DESARROLLO, 10(26). https:// doi.org/10.31644/IMASD.26.2021.a03

\section{RESUMEN}

Se estudió el comportamiento de diferentes densidades de siembra de dedinios $\left(40,60,80\right.$ y 100 peces $/ \mathrm{m}^{2}$ ) para el engorde de trucha Arco Iris (Oncorhynchus mykiss); los peces empleados fueron 840 dedinios de 4 meses de edad y con un peso promedio de $24,20 \mathrm{gr}$; y una talla promedio de $2,92 \mathrm{~cm}$ de longitud total del cuerpo; los mismos que se distribuyeron en un diseño en bloques completamente al azar con tres repeticiones por tratamiento, teniendo un total de 12 unidades experimentales. Los pesos promedios al inicio de la investigación fueron de 24,20 gr y transcurridos 7 meses fueron de 253,75 gr. Mientras que el consumo total de alimento fue de 316,81 gr durante los 7 meses de evaluación. Al mismo tiempo los peces ganaron 229,55 gramos en total. Las truchas resultaron buenas convertidoras de alimento puesto que requirieron entre 1,11 y 1,71 kilos de balanceado para convertir un kilo de carne. En referencia a la talla no existieron diferencias estadísticas significativas en los tratamientos, iniciando con $12,92 \mathrm{~cm}$ y finalmente llegaron a una longitud total del cuerpo de $26,95 \mathrm{~cm}$ en la última evaluación (séptimo mes).

\section{Palabras clave}

Dedinios; Trucha Arco Iris. 


\section{- Abstract-}

The behavior of different planting densities of dedinios (40, 60, 80 and 100 fish $/ \mathrm{m}^{2}$ ) was studied for the fattening of Rainbow trout (Oncorhynchus mykiss); the fish used were 840 dedinios of 4 months of age and with an average weight of $24.20 \mathrm{gr}$; and an average size of $2.92 \mathrm{~cm}$ in total body length; the same ones that were distributed in a completely randomized block design with three replications per treatment, having a total of 12 experimental units. The average weights at the beginning of the investigation were $24.20 \mathrm{gr}$ and after 7 months they were $253.75 \mathrm{gr}$. While the total food consumption was 316.81 gr during the 7 months of evaluation. At the same time the fish gained 229.55 grams in total. Trout were good food converters since they required between 1.11 and 1.71 kilos of balanced to convert one kilo of meat. Regarding height, there were no significant statistical differences in the treatments, starting with $12.92 \mathrm{~cm}$ and finally reaching a total body length of $26.95 \mathrm{~cm}$ in the last evaluation (seventh month).

\section{Keywords}

Dedinios; Rainbow trout. 
L. a trucha Arco Iris (Oncorhynchus mykiss) es nativa de las cuencas que drenan al Pacífico en Norte América, abarcando desde Alaska a México. Desde 1874 ha sido introducida en las aguas de todos los continentes excepto la Antártica, con propósitos recreacionales para pesca deportiva y para acuicultura (FAO, 2020).

La trucha es una especie anádroma, pero también sedentaria (no migratoria). En libertad, vive en ríos de montaña con agua fría (Flores, 2018). La república del Ecuador cuenta con una diversidad de climas que incentivan a la realización de actividades productivas agrícolas y acuícolas. Es catalogado como país agropecuario por ser productor de banano, flores y camarones. El cultivo de la trucha arco iris es una opción para incentivar, debido a las condiciones favorables que brinda el país. Esta actividad se ha desarrollado en la región interandina porque cuenta con temperaturas del agua entre $5^{\circ} \mathrm{C}$ y $18^{\circ} \mathrm{C}$ y poseen mayor recurso hídrico (Gallardo Domínguez, 2015).

Imaki (2003) indica que a partir de 1930 se construyeron salas de incubación y alevinaje en las provincias de Imbabura, Cotopaxi y Azuay respectivamente, con la introducción de huevos embrionados de trucha cuyos alevines sirvieron para poblar los sistemas fluviales y lacustres de la región Interandina.

La provincia de Chimborazo presenta en su diversidad ecográfica una gran potencialidad de recurso hídrico en los diferentes pisos altitudinales, haciendo que la piscicultura pueda desarrollarse con grandes ventajas productivas y económicas; es así que según manifiesta el Ministerio de Agricultura, Ganadería, Acuacultura y Pesca (MAGAP) en los últimos años se ha incrementado sustancialmente este tipo de emprendimiento (Morales, 2019).

La trucha es un pez exigente en la calidad y la cantidad de agua, se crían en aguas limpias, frías y bien oxigenadas (Calle \& Calle 2017). En este contexto, el uso eficiente de alimentos reduciría los de costos de producción y tendría un impacto importante en la rentabilidad, principalmente en los sistemas de producción de escala avanzada y de mayor escala (Carpio \& Tito, 2017). Con esta investigación se pretende optimizar el espacio útil para la cría de truchas, mediante la utilización de una adecuada densidad de peces por metro cuadrado, ya que se conoce que en nuestro país este parámetro no es tomado en cuenta como criterio técnico.

\section{MATERIALES Y MÉTODOS}

\section{Localización y duración del experimento}

La presente investigación se realizó en el programa de producción piscícola del colegio Nacional Técnico Autachi, de la comunidad Nitiluisa, parroquia Calpi, cantón Riobamba, provincia de Chimborazo, Ecuador. Este experimento 
duró 270 días, distribuidos en 30 días para la preparación de estanques y adaptación de los peces; 210 días para la producción de truchas y 30 días para la tabulación e interpretación de datos y publicación.

\section{Unidades experimentales}

En la presente investigación se trabajó con 840 dedinios, para lo cual se utilizaron 12 unidades experimentales (estanques) de $1 \mathrm{~m}^{2}$ por $1 \mathrm{~m}$ de profundidad, en los cuales se distribuyeron los respectivos tratamientos (40, 60, 80 y 100 dedinios $/ \mathrm{m}^{2}$ ).

\section{Materiales}

12 estanques de $1 \mathrm{~m}^{2}, 840$ dedinios, alimento balanceado, termómetro, cinta métrica, atarrayas, material de escritorio, challos o nasas, azul de metileno, antibióticos, formol, cloro, sal común, hipoclorito de sodio e insumos de limpieza necesarios.

\section{Equipos}

Balanza de precisión, cámara fotográfica, calculadora, hictiometro, peachimetro, termómetro.

\section{Instalaciones}

Estanques de engorde.

Tratamiento y diseño del experimento

Los tratamientos que se aplicaron en esta investigación fueron: T1: 40 dedinios $/ \mathrm{m}^{2}$, T2: 60 dedinios $/ \mathrm{m}^{2}$, T3: 80 dedinios $/ \mathrm{m}^{2}$, T4: 100 dedinios $/ \mathrm{m}^{2}$. Los mismos fueron distribuidos en un Diseño de Bloques Completamente al Azar (DBCA).

\section{Análisis estadístico}

Los resultados del presente estudio fueron sometidos a los siguientes análisis estadísticos: Análisis de varianza (ADEVA), Prueba de separación de medias (Duncan, $\alpha \leq 0.05$ ), Análisis de regresión lineal. 


\section{Procedimiento experimental}

Antes del inicio de la investigación se prepararon y desinfectaron los estanques para conformar las unidades experimentales de $1 \mathrm{~m}^{2}$, se distribuyeron los peces de acuerdo a su peso en cada uno de los bloques, se sorteó o se distribuyeron al azar los tratamientos. Todas las unidades experimentales tuvieron igual tratamiento y manejo alimenticio, el suministro del mismo se realizó dos veces durante el día.

El peso de los peces se tomó quincenalmente para calcular la ganancia de peso mediante el análisis de la varianza, de igual manera la conversión alimenticia, que se calculó con el consumo de alimento. Se tomaron datos del tamaño a los 15 días y al final del experimento, el costo del alimento por kilogramo de ganancia de peso al final de la fase de engorde; así como el Beneficio/costo.

A los peces muertos se realizó la respectiva necropsia para determinar la causa de la muerte, se tomó el porcentaje de mortalidad semanal y final. Al final del experimento se pesó al 30\% de los dedinios para hacer el análisis del incremento de peso desde el inicio al final de la fase de engorde.

\section{Programa sanitario}

Quince días antes de comenzar el experimento se limpiaron los estanques y se desinfectó con formol; y 3 días antes de la llegada de los dedinios se procedió a desinfectarlos con azul de metileno, los peces pasaron una etapa de adaptación de 15 días para luego iniciar con el experimento, para lo cual se les colocó de acuerdo con el sorteo de los tratamientos (40, 60, 80 y 100 dedinios $/ \mathrm{m}^{2}$ ), y se tomaron datos de peso y tamaño inicial. Con el transcurso de la investigación se siguió evaluando las variables de estudio, para determinar el beneficio/costo. La cantidad de alimento suministrado se calculó de acuerdo con el número de peces, peso, tamaño y temperatura del agua de acuerdo con las recomendaciones de la casa comercial.

La limpieza de los estanques se realizó una vez por semana. Se utilizó como desinfectante Formol y Cloro, además se utilizó Hipoclorito de Sodio para la neutralización del Cloro.

\section{RESULTADOS Y DISCUSIÓN}

\section{Evaluación del peso}

En la evaluación a los 210 días en los diferentes tratamientos utilizados no presentaron diferencias estadísticas significativas, aunque los pesos numéricamente se situaron en el siguiente orden: 245,93 gr los 40 peces $/ \mathrm{m}^{2} ; 258,68$ 
gr los 60 peces $/ \mathrm{m}^{2} ; 257,72$ gr los 80 peces $/ \mathrm{m}^{2} ;$ y 252,65 gr los 100 peces $/ \mathrm{m}^{2}$ (Tabla 1), Esto probó finalmente que la variable peso no se vio afectada por la densidad de animales por metro cuadrado.

De este análisis se dedujo que estadísticamente el peso no fue influyente en las densidades de peces utilizadas para la investigación; particular que es ventajoso, puesto que en la mayoría de los casos, tradicionalmente en los estanques de cemento armado, en la sierra ecuatoriana se viene utilizando densidades de máximo 70 peces $/ \mathrm{m}^{2}$, mientras que si existe la suficiente dosificación de agua se podrían incluir 100 peces $/ \mathrm{m}^{2}$ (Blanco, 1995) y (Stevenson, 1985). De manera similar Imaki (2003) indica que, si las condiciones de suministro de agua son favorables, los estanques pueden entregar hasta 25 kilos de peces $/ \mathrm{m}^{2}$ de superficie, si consideramos que cada animal puede llegar con 250 gr al "tipo plato", estaríamos hablando de 100 peces $/ \mathrm{m}^{2}$.

\section{Tabla 1}

Comportamiento del peso en el engorde de truchas mediante diferentes densidades de peces

\begin{tabular}{|c|c|c|c|c|c|c|c|}
\hline \multirow{2}{*}{$\begin{array}{l}\text { VARIABLE } \\
\text { Peso }\end{array}$} & \multicolumn{4}{|c|}{ TRATAMIENTOS } & \multirow{2}{*}{ MEDIA } & \multirow{2}{*}{$\begin{array}{l}\text { CV } \\
(\%)\end{array}$} & \\
\hline & 40 peces $/ \mathrm{m}^{2}$ & 60 peces $/ \mathrm{m}^{2}$ & 80 peces $/ \mathrm{m}^{2}$ & 100 peces $/ \mathrm{m}^{2}$ & & & \\
\hline Inicial (gr) & $24,95 \mathrm{~A}$ & $24,02 \mathrm{~A}$ & $24,02 \mathrm{~A}$ & $21,73 \mathrm{~A}$ & 24.20 & 30.76 & ns \\
\hline A los 30 días (gr) & $43,43 \mathrm{~A}$ & $47,48 \mathrm{~A}$ & $47,48 \mathrm{~A}$ & $38,73 \mathrm{~A}$ & 44,98 & 22.26 & ns \\
\hline A los 60 días (gr) & $66,043 \mathrm{~A}$ & $70,3 \mathrm{~A}$ & $70,3 \mathrm{~A}$ & $61,673 \mathrm{~A}$ & 65,958 & 20.89 & ns \\
\hline A los 90 días (gr) & $99,05 \mathrm{~A}$ & $89,767 \mathrm{~A}$ & $89,767 \mathrm{~A}$ & $80,237 \mathrm{~A}$ & 89,741 & 19.34 & ns \\
\hline A los 120 días (gr) & $142,05 \mathrm{~A}$ & $138,13 \mathrm{~A}$ & $138,13 \mathrm{~A}$ & $132,59 \mathrm{~A}$ & 138,45 & 10.62 & ns \\
\hline A los 150 días (gr) & $175,52 \mathrm{~A}$ & $170,64 \mathrm{~A}$ & $170,64 \mathrm{~A}$ & $165,32 \mathrm{~A}$ & 171,38 & 9.19 & ns \\
\hline A los 180 días (gr) & $208,98 \mathrm{~A}$ & 203,39 A & $203,39 \mathrm{~A}$ & $198,07 \mathrm{~A}$ & 204,37 & 8.24 & ns \\
\hline A los 210 días (gr) & $245,93 \mathrm{~A}$ & $257,72 \mathrm{~A}$ & $257,72 \mathrm{~A}$ & $252,65 \mathrm{~A}$ & 253,75 & 4.89 & ns \\
\hline
\end{tabular}

CV: Coeficiente de variación ns: No existen diferencias estadísticas significativas $(P>0,05)$

Promedios con letras iguales no difieren estadísticamente según la prueba de Duncan

Fuente: elaboración propia

\section{Evaluación del consumo de alimento}

En la última evaluación a los 210 días, los diferentes tratamientos aplicados no presentaron diferencias estadísticas significativas, sin embargo, el consumo de alimento en forma numérica se escalono en el siguiente orden: 68,963 gr de 40 peces $/ \mathrm{m}^{2} ; 68,325$ gr de 60 peces $/ \mathrm{m}^{2} ; 67,119$ gr de 80 peces $/ \mathrm{m}^{2}$ y 65,362 gr de 100 peces $/ \mathrm{m}^{2}$ (Tabla 2 ). Esto nos experimentaría que hasta el final de la evaluación la variable consumo de alimento no se afecta por los diferentes tratamientos aplicados. 


\section{Tabla 2}

Consumo de alimento en el engorde de truchas mediante diferentes densidades de peces

\begin{tabular}{|c|c|c|c|c|c|c|c|}
\hline \multirow{2}{*}{$\begin{array}{c}\text { VARIABLE } \\
\text { Consumo de } \\
\text { alimento (gr) }\end{array}$} & \multicolumn{4}{|c|}{ TRATAMIENTOS } & \multirow{2}{*}{ MEDIA } & \multirow{2}{*}{$\begin{array}{l}\text { CV } \\
\text { (\%) }\end{array}$} & \\
\hline & 40 peces $/ \mathrm{m}^{2}$ & 60 peces $/ \mathrm{m}^{2}$ & 80 peces $/ m^{2}$ & 100 peces $/ \mathrm{m}^{2}$ & & & \\
\hline A los 30 días & $26,946 \mathrm{~A}$ & $28,188 \mathrm{~A}$ & $25,942 \mathrm{~A}$ & $23,472 \mathrm{~A}$ & 26,137 & 30.77 & ns \\
\hline A los 60 días & $25,727 \mathrm{~A}$ & $25,645 \mathrm{~A}$ & $29,959 \mathrm{~A}$ & $24,976 \mathrm{~A}$ & 26,576 & 22.02 & ns \\
\hline A los 90 días & $42,882 \mathrm{~A}$ & $33,567 \mathrm{~A}$ & $35,853 \mathrm{~A}$ & $30,477 \mathrm{~A}$ & 35,695 & 17.87 & ns \\
\hline A los 120 días & $48,204 \mathrm{~A}$ & $57,172 \mathrm{~A}$ & $54,134 \mathrm{~A}$ & $57,134 \mathrm{~A}$ & 54,161 & 14.86 & ns \\
\hline A los 150 días & $49,258 \mathrm{~A}$ & $46,544 \mathrm{~A}$ & $58,894 \mathrm{~A}$ & $46,277 \mathrm{~A}$ & 50,243 & 26.70 & ns \\
\hline A los 180 días & $57,921 \mathrm{~A}$ & $57,435 \mathrm{~A}$ & $56,312 \mathrm{~A}$ & $54,557 \mathrm{~A}$ & 56,556 & 9.19 & ns \\
\hline A los 210 días & $68,963 \mathrm{~A}$ & $68,325 \mathrm{~A}$ & $67,119 \mathrm{~A}$ & $65,362 \mathrm{~A}$ & 67,442 & 8.24 & ns \\
\hline Total & 319,9 A & $316,88 \mathrm{~A}$ & $328,21 \mathrm{~A}$ & $302,25 \mathrm{~A}$ & 316,81 & 9.48 & ns \\
\hline
\end{tabular}

CV: Coeficiente de variación

ns: No existen diferencias estadísticas significativas $(P>0,05)$

Promedios con letras iguales no difieren estadísticamente según la prueba de Duncan

Fuente: elaboración propia

De acuerdo con el análisis estadístico, se determinó que la densidad de peces por metro cuadrado no es influyente en el engorde de trucha Arco Iris, es decir que el consumo es relativamente normal con o sin hacinamiento, por lo cual es posible un manejo sostenido de la estanquería de truchas, siempre y cuando los caudales sean precisos. Según la FAO (2014) la cantidad de alimento que se debe suministrar a cada grupo de truchas depende de la clase de alimento, la calidad del agua, temperatura, estado y tamaño de los peces. Del mismo modo, Gómez (2017) menciona que el alimento se debe distribuir al boleo (esparciéndolo al aire), de acuerdo con la ración diaria que corresponde a cada uno de los estanques, para asegurar un crecimiento uniforme.

Evaluación de la ganancia de peso

A los 210 días que se realizado la última evaluación, los diferentes tratamientos aplicados no mostraron diferencias estadísticas significativas, en tal virtud el parámetro ganancia de peso se escalono así: 54,583 gr de 100 peces $/ \mathrm{m}^{2}$; 54,327 gr de 80 peces $/ \mathrm{m}^{2} ; 51,637$ gr de 60 peces $/ \mathrm{m}^{2}$ y 36,953 gr de 40 peces/ $\mathrm{m}^{2}$ (Tabla 3). Esto nos demuestra que hasta la última evaluación la variable ganancia de peso estadísticamente no demostró ser significativa debido a los tratamientos. 
Tabla 3

Ganancia de peso en el engorde de truchas mediante diferentes densidades de peces

\begin{tabular}{|c|c|c|c|c|c|c|c|}
\hline \multirow{2}{*}{$\begin{array}{c}\text { VARIABLE } \\
\text { Ganancia de peso } \\
\text { (gr) }\end{array}$} & \multicolumn{4}{|c|}{ TRATAMIENTOS } & \multirow[b]{2}{*}{ MEDIA } & \multirow{2}{*}{$\begin{array}{l}\text { CV } \\
\text { (\%) }\end{array}$} & \\
\hline & 40 peces $/ \mathrm{m}^{2}$ & 60 peces $/ \mathrm{m}^{2}$ & 80 peces $/ \mathrm{m}^{2}$ & 100 peces $/ \mathrm{m}^{2}$ & & & \\
\hline A los 30 días & $18,483 \mathrm{~A}$ & $24,183 \mathrm{~A}$ & $23,463 \mathrm{~A}$ & $17 \mathrm{~A}$ & 20,783 & 15.87 & ns \\
\hline A los 60 días & $22,61 \mathrm{~A}$ & $15,533 \mathrm{~A}$ & $22,817 \mathrm{~A}$ & $22,94 \mathrm{~A}$ & 20,975 & 28.37 & ns \\
\hline A los 90 días & $33,007 \mathrm{~A}$ & $24,093 \mathrm{~A}$ & $19,467 \mathrm{~A}$ & $18,563 \mathrm{~A}$ & 23,783 & 30.81 & ns \\
\hline A los 120 días & $43,00 \mathrm{~A}$ & $51,133 \mathrm{~A}$ & $48,367 \mathrm{~A}$ & $52,35 \mathrm{~A}$ & 48,713 & 14.77 & ns \\
\hline A los 150 días & $33,467 \mathrm{~A}$ & $33,003 \mathrm{~A}$ & $32,51 \mathrm{~A}$ & $32,737 \mathrm{~A}$ & 32,929 & 4.15 & ns \\
\hline A los 180 días & $33,463 \mathrm{~A}$ & $33,00 \mathrm{~A}$ & $32,747 \mathrm{~A}$ & $32,743 \mathrm{~A}$ & 32,988 & 4.08 & ns \\
\hline A los 210 días & $36,953 \mathrm{AB}$ & $51,637 \mathrm{AB}$ & $54,327 \mathrm{~A}$ & $54,583 \mathrm{~A}$ & 49,375 & 13.30 & * \\
\hline Total & $220,98 \mathrm{~A}$ & $232,58 \mathrm{~A}$ & $233,7 \mathrm{~A}$ & $230,92 \mathrm{~A}$ & 229,55 & 2.89 & ns \\
\hline
\end{tabular}

CV: Coeficiente de variación

ns: No existen diferencias estadísticas significativas $(P>0,05)$

*: Diferencias significativas $(P \leq 0,05)$

Promedios con letras iguales no difieren estadísticamente según la prueba de Duncan

Fuente: elaboración propia

Evidentemente en la presente investigación se da cuenta que la ganancia de peso en cada evaluación, e inclusive en el período total, no tuvo influencia estadística sobre el manejo de las diferentes densidades de peces. Al respecto Blanco (1995) y Imaki (2003) mencionan que basta que exista un caudal aceptable y completa profilaxis, se puede mantener hasta 100 peces de 250 gramos $/ \mathrm{m}^{2}$ o también 25 kilos de trucha tipo plato $/ \mathrm{m}^{2}$ de estanque; sin que se interfiera en la ganancia de peso.

\section{Evaluación de la conversión alimenticia}

Al final de la evaluación a los 210 días, los cuatro tratamientos utilizados no presentaron diferencias estadísticas significativas, sin embargo los consumos se situaron en el siguiente orden: 2,08 de conversión en la unidad experimental que contenía 40 peces $/ \mathrm{m}^{2} ; 1,33$ de conversión alimenticia por los 60 peces $/ \mathrm{m}^{2}$ de superficie; 1,24 gramos de balanceado aprovechados para ganar un gramo de carne por parte de los animales, soportando una densidad de 80 peces $/ \mathrm{m}^{2}$ y 1,2 de conversión en peces incluidos a razón de 100 peces $/ \mathrm{m}^{2}$ (Tabla 4 ). Esto indicó que hasta el final del experimento la conversión alimenticia no se vio afectada por la densidad de animales bajo los tratamientos detallados. 
Tabla 4

Conversión de peso en el engorde de truchas mediante diferentes densidades de peces

\begin{tabular}{|c|c|c|c|c|c|c|c|}
\hline \multirow{2}{*}{$\begin{array}{l}\text { VARIABLE } \\
\text { Conversión } \\
\text { alimenticia }\end{array}$} & \multicolumn{4}{|c|}{ TRATAMIENTOS } & \multirow[b]{2}{*}{ MEDIA } & \multirow{2}{*}{$\begin{array}{l}\text { CV } \\
(\%)\end{array}$} & \\
\hline & 40 peces $/ \mathrm{m}^{2}$ & 60 peces $/ \mathrm{m}^{2}$ & 80 peces $/ m^{2}$ & 100 peces $/ \mathrm{m}^{2}$ & & & \\
\hline A los 30 días & $1,41 \mathrm{~A}$ & $1,16 \mathrm{~A}$ & $1,11 \mathrm{~A}$ & $1,40 \mathrm{~A}$ & 1,27 & 25,23 & ns \\
\hline A los 60 días & $1,15 \mathrm{~B}$ & $1,73 \mathrm{~A}$ & $1,32 \mathrm{AB}$ & $1,09 \mathrm{~B}$ & 1,32 & 12,50 & $* *$ \\
\hline A los 90 días & $1,36 \mathrm{~A}$ & $1,39 \mathrm{~A}$ & $1,98 \mathrm{~A}$ & $1,71 \mathrm{~A}$ & 1,61 & 25,47 & ns \\
\hline A los 120 días & $1,12 \mathrm{~A}$ & $1,12 \mathrm{~A}$ & $1,12 \mathrm{~A}$ & $1,09 \mathrm{~A}$ & 1,11 & 1,76 & ns \\
\hline A los 150 días & $1,47 \mathrm{~A}$ & $1,41 \mathrm{~A}$ & $1,80 \mathrm{~A}$ & $1,41 \mathrm{~A}$ & 1,52 & 25,54 & ns \\
\hline A los 180 días & $1,72 \mathrm{~A}$ & $1,74 \mathrm{~A}$ & $1,72 \mathrm{~A}$ & $1,67 \mathrm{~A}$ & 1,71 & 6,39 & ns \\
\hline A los 210 días & $2,09 \mathrm{~A}$ & $1,33 \mathrm{~A}$ & $1,24 \mathrm{~A}$ & $1,20 \mathrm{~A}$ & 1,46 & 37.39 & ns \\
\hline Total & $1,44 \mathrm{~A}$ & $1,36 \mathrm{~A}$ & $1,40 \mathrm{~A}$ & $1,31 \mathrm{~A}$ & 1,38 & 7.82 & ns \\
\hline
\end{tabular}

CV: Coeficiente de variación

ns: No existen diferencias estadísticas significativas $(P>0,05)$

**: Diferencias altamente significativas $(\mathrm{P} \leq 0,01)$

Promedios con letras iguales no difieren estadísticamente según la prueba de Duncan

Fuente: elaboración propia

Este análisis indica que todas las unidades experimentales tuvieron la misma actitud para convertir alimento, entonces el incremento en la densidad de peces no fue influyente en el engorde de trucha Arco Iris, lo que puede indicar que en las condiciones climáticas de la provincia de Chimborazo, Ecuador, se podría mantener hasta 100 peces $/ \mathrm{m}^{2}$ o más dependiendo de los estanques, sin que se alteren los consumos, pesos y conversiones de alimento, desde luego manteniendo las condiciones óptimas del agua (cantidad y calidad) y temperatura ambiental adecuada

Colque (2020) menciona que se puede mantener hasta 19,62 kg/ $\mathrm{m}^{2}$, equivalente a 80 peces por $\mathrm{m}^{2}$ de superficie; superado ampliamente a lo manifestado por Calle \& Calle (2017) donde indican que en el proceso de producción de truchas arcoíris producidas a base de alimentos naturales (cereales ecológicos y harina de pescado) se puede albergar de 25 a 30 truchas $/ \mathrm{m}^{2}$.

Del mismo modo Morales (2004) en su investigación sobre el crecimiento y eficiencia alimentaria de trucha arco iris en jaulas, manifiesta que la ración óptima diaria encontrada correspondió al 2.9\% del peso corporal.

\section{Evaluación de la talla}

Al final de la investigación, a los 210 días la variable talla, los 4 tratamientos utilizados no mostraron diferencias estadísticas significativas, aunque estas tallas numéricamente se mostraron en el siguiente orden de tratamientos: $27,35 \mathrm{~cm}$ por parte de los 60 peces estabulados en un metro cuadrado; 27,28 
cm de longitud de los peces que soportaron una densidad de 80 por unidad de superficie; 26,93 cm de longitud total del cuerpo de los peces que resistieron una carga animal de 100 por unidad de superficie y 26,25 centímetros las truchas con una densidad de siembra de 80 por metro cuadrado (Tabla 5). Esta evaluación nos indica que, hasta el final, la talla no es diferenciada por parte de los tratamientos utilizados.

\section{Tabla 5}

Comportamiento de la talla en el engorde de truchas mediante diferentes densidades de peces

\begin{tabular}{|c|c|c|c|c|c|c|c|}
\hline \multirow{2}{*}{$\begin{array}{l}\text { VARIABLE } \\
\text { Talla }\end{array}$} & \multicolumn{4}{|c|}{ TRATAMIENTOS } & \multirow{2}{*}{ MEDIA } & \multirow{2}{*}{$\begin{array}{l}\text { CV } \\
(\%)\end{array}$} & \\
\hline & 40 peces $/ \mathrm{m}^{2}$ & 60 peces $/ \mathrm{m}^{2}$ & 80 peces $/ m^{2}$ & 100 peces $/ \mathrm{m}^{2}$ & & & \\
\hline Inicial (cm) & $12,97 \mathrm{~A}$ & $13,42 \mathrm{~A}$ & $13,17 \mathrm{~A}$ & $12,15 \mathrm{~A}$ & 12,93 & 14,28 & ns \\
\hline A los 30 días $(\mathrm{cm})$ & $15,42 \mathrm{~A}$ & $15,87 \mathrm{~A}$ & $14,77 \mathrm{~A}$ & $14,30 \mathrm{~A}$ & 15,09 & 10,12 & ns \\
\hline A los 60 días $(\mathrm{cm})$ & $16,82 \mathrm{~A}$ & $17,05 \mathrm{~A}$ & $16,65 \mathrm{~A}$ & $16,44 \mathrm{~A}$ & 16,74 & 8,45 & ns \\
\hline A los 90 días $(\mathrm{cm})$ & $18,49 \mathrm{~A}$ & $18,55 \mathrm{~A}$ & $17,85 \mathrm{~A}$ & $18,06 \mathrm{~A}$ & 18,24 & 8,42 & ns \\
\hline A los 120 días $(\mathrm{cm})$ & $20,54 \mathrm{~A}$ & $21,00 \mathrm{~A}$ & $20,52 \mathrm{~A}$ & $20,42 \mathrm{~A}$ & 20,62 & 6,43 & ns \\
\hline A los 150 días (cm) & $22,37 \mathrm{~A}$ & $22,89 \mathrm{~A}$ & $22,51 \mathrm{~A}$ & $22,51 \mathrm{~A}$ & 22,57 & 5,53 & ns \\
\hline A los 180 días $(\mathrm{cm})$ & $24,21 \mathrm{~A}$ & $24,79 \mathrm{~A}$ & $24,49 \mathrm{~A}$ & $24,60 \mathrm{~A}$ & 24,52 & 4,82 & ns \\
\hline A los 210 días $(\mathrm{cm})$ & $26,25 \mathrm{~A}$ & $27,35 \mathrm{~A}$ & $27,28 \mathrm{~A}$ & $26,93 \mathrm{~A}$ & 26,95 & 4,03 & ns \\
\hline
\end{tabular}

CV: Coeficiente de variación

ns: No existen diferencias estadísticas significativas $(P>0,05)$

Promedios con letras iguales no difieren estadísticamente según la prueba de Duncan

Fuente: elaboración propia

En esta investigación se demuestra que la talla no es influyente sobre la densidad de peces y el crecimiento de truchas Arco Iris. Según Bustamante, Araníbar, Huanca, \& Rodriguez (2016) y FAO (2014) el factor más importante que regula el crecimiento de los peces es la temperatura del agua, ya que estos no tienen capacidad propia para regular su temperatura corporal. A mayor temperatura del agua corresponde una mayor tasa metabólica.

La especie puede soportar amplias gamas de variación de temperatura $\left(0-27{ }^{\circ} \mathrm{C}\right)$, pero el desove y crecimiento ocurren en una gama más estrecha (9-14 $\left.{ }^{\circ} \mathrm{C}\right)$. La temperatura óptima del agua para el cultivo de trucha Arco Iris (Oncorhynchus mykiss) está por debajo de $21^{\circ} \mathrm{C}$ (FAO, 2020). Es así que en la presente investigación con $10-11^{\circ} \mathrm{C}$ de temperatura del agua en promedio, se engordaron truchas tipo plato ( $250 \mathrm{gr}$ ), en 7 meses (desde dedinio a tamaño comercial); mientras que en aguas con $16^{\circ} \mathrm{C}$ de promedio se obtendrían peces de la misma condición productiva en 3 o 4 meses; por lo tanto es conveniente seguir investigando el efecto de la temperatura del agua y las condiciones climáticas en el desarrollo de la trucha, puesto que los 
peces son animales poiquilotermos y dependen de la temperatura ambiental para su metabolismo.

\section{Mortalidad}

En el Tabla 6 se encuentran los parámetros de mortalidad que se pudo presentar en el engorde de Trucha Arco Iris bajo diferentes densidades de cría.

\section{Tabla 6}

Mortalidad de peces criados en diferentes densidades

\begin{tabular}{ccc}
\hline Tratamientos & \multicolumn{3}{c}{ Mortalidad } \\
& $N^{\circ}$ & $\%$ \\
40 Peces $/ \mathrm{m}^{2}$ & 1 & 0,12 \\
60 Peces $/ \mathrm{m}^{2}$ & 1 & 0,12 \\
80 Peces $/ \mathrm{m}^{2}$ & 5 & 0,60 \\
100 Peces $/ \mathrm{m}^{2}$ & 7 & 0,83 \\
Total & 14 & 1,67 \\
\hline
\end{tabular}

Fuente: elaboración propia

Se puede advertir que la mortalidad total fue de 14 ejemplares de 840 , es decir $1,67 \%$, de los cuales las unidades experimentales en donde se albergaron 100 truchas $/ \mathrm{m}^{2}$ fueron las que más alta tasa alcanzaron $(0,83 \%)$, no obstante, Zapata (2015) manifiesta que la baja mortalidad es debido a que no existió exceso de estrés en los peces y se mantuvo los estanques limpios y protegidos de depredadores.

Del mismo modo Zambrano (2013) menciona que el porcentaje de mortalidad durante todo el proceso de crecimiento se ha calculado por fases: en la etapa de dedinaje un $6,50 \%$, levante $3,60 \%$ y engorde $5,40 \%$ en total $15,50 \%$ durante nueve meses en estanques de hormigón, parámetro evidentemente superior a nuestros datos. Con este particular es por demás ventajoso contar con una buena alternativa tecnológica para emprender la piscicultura en la sierra Ecuatoriana.

\section{Evaluación económica}

Según este análisis, podemos indicar que a medida que se incrementa la densidad de peces en los estanques, también los ingresos netos suben desde 0,75 dólares con 40 peces $/ \mathrm{m}^{2} ; 3,62$ dólares con 60 peces $/ \mathrm{m}^{2} ; 5,11$ dólares con 80 peces $/ \mathrm{m}^{2}$; hasta 8,68 dólares en truchas que se mantuvieron a razón de $100 / \mathrm{m}^{2}$ (Tabla 7 ). 
Igualmente, el Beneficio/Costo, aunque es positivo en todos los tratamientos, siempre la mayor densidad (100 peces $/ \mathrm{m}^{2}$ ) obtuvo el indicador más óptimo, puesto que, por cada unidad monetaria invertida, se obtiene 16 centavos de ganancia o 16\% de rentabilidad en 7 meses de ejercicio productivo-económico.

\section{Tabla 7}

Análisis económico de truchas criadas bajo diferentes densidades

\begin{tabular}{|c|c|c|c|c|c|c|c|}
\hline \multirow{2}{*}{ DETALLE } & \multirow{2}{*}{ Unidad } & \multirow{2}{*}{ Cant. } & \multirow{2}{*}{ VU } & \multicolumn{4}{|c|}{ TRATAMIENTOS (TRUCHAS / $\mathrm{M}^{2}$ ) } \\
\hline & & & & 40 & 60 & 80 & 100 \\
\hline Estanques (depreciación) & $m^{2}$ & 1 & 2,33 & 2,33 & 2,33 & 2,33 & 2,33 \\
\hline Divisiones (malla) - deprec. & u & 1 & 0,23 & 0,23 & 0,23 & 0,23 & 0,23 \\
\hline Insumos & u & 1 & - & 0,20 & 0,30 & 0,40 & 0,50 \\
\hline Medicinas & u & 1 & - & 0,10 & 0,15 & 0,20 & 0,25 \\
\hline Alimentación (según consumo) & $\mathrm{kg}$ & - & 0,72 & 9,21 & 13,69 & 18,90 & 21,76 \\
\hline Mano de Obra (3 Jorn/mes) & jornal & - & 4 & 11,76 & 18,48 & 24,36 & 29,4 \\
\hline TOTAL DE GASTOS & & & & 23,84 & 35,19 & 46,43 & 54,48 \\
\hline Peso Promedio & $\mathrm{Kg} / \mathrm{pez}$ & & & 0,25 & 0,26 & 0,26 & 0,25 \\
\hline Peso / Unidad Experimental & $\mathrm{Kg}$ & & & 9,84 & 15,52 & 20,62 & 25,27 \\
\hline TOTAL INGRESOS BRUTOS & $\$$ & 1 & $2,5 / \mathrm{Kg}$ & 24,59 & 38,80 & 51,54 & 63,16 \\
\hline INGRESOS NETOS & & & & 0,75 & 3,62 & 5,11 & 8,68 \\
\hline BENEFICIO / COSTO & & & & 1,03 & 1,10 & 1,11 & 1,16 \\
\hline
\end{tabular}

Cant: Cantidad

VU: Valor Unitario

Fuente: elaboración propia

\section{CONCLUSIONES Y RECOMENDACIONES}

En toda la investigación no se descubrió diferencia estadística significativa en las diferentes variables, es decir que conviene criar hasta 100 peces $/ \mathrm{m}^{2}$, siempre y cuando existan condiciones óptimas del agua (cantidad y calidad) y temperatura ambiental adecuada.

Los pesos de las truchas en promedio fueron 44,$98 ; 65,95 ; 89,74 ; 138,45$; 171,38; 204,37 y, 253,75 gramos desde el primer al séptimo mes de evaluación respectivamente. Las truchas resultaron buenas convertidoras de alimento puesto que requirieron entre 1,11 y 1,71 kilos de balanceado para convertir un kilo de carne.

En referencia a la talla, igualmente no existieron diferencias estadísticas en los tratamientos, aunque los promedios generales fueron de: 15,09 centímetros el primer mes, 16,74 cm el segundo, 18,24 cm el tercer mes, 20,62 centímetros 
en la cuarta evaluación, 22,57 cm en el quinto mes, $24,52 \mathrm{~cm}$ en el sexto mes y finalmente 26,95 centímetros en la última evaluación (séptimo mes).

De acuerdo con el análisis económico se puede recomendar el manejo de hasta 100 peces por metro cuadrado de superficie de estanque para la cría de trucha Arco Iris en la sierra Ecuatoriana, dependiendo de la disponibilidad de agua (caudal) y de su calidad, puesto que con este particular se optimiza el espacio y la inversión. 


\section{REFERENCIAS}

Blanco, C. (1995). La trucha cría industrial. Barcelona, España: Editorial S.A. MUNDI-PRENSA LIBROS.

Bustamante, L., Araníbar, A., Huanca, B., \& Rodriguez, F. (2016). Determinación de índices productivos de truchas arco iris (Oncorhynchus mykiss) alimentadas con dietas orgánicas en fase de engorde. Revista Ciencia Animal, 1(2), 91-97.

Calle, O., \& Calle, A. (2017). Producción de trucha arco iris a base a alimentos orgánicos. Repositorio digital Universidad de Mayor de San Andrés. Bolivia.

Carpio, E., \& Tito, E. (2017). Escalas productivas y nivel de riesgo del productor de trucha, puno-Perú.COMUNI@CCIÓN: Revista de Investigación en Comunicación y Desarrollo, 8(2), 81-93.

Colque, R. (2020). Determinación del factor de conversión alimentaria y crecimiento de trucha arco iris (Oncorhynchus mykiss) bajo diferentes densidades y niveles de alimentación en estanques artificiales en Llaullini , La Paz. 6(1), 1820-1838.

FAO. (2020). Programa de información de especies acuáticas. http://www.fao. org/fishery/culturedspecies/Oncorhynchus_mykiss/es

FAO. (2014). Manual práctico para el cultivo de la trucha arcoíris. Guatemala. http://www.fao.org/3/a-bc354s.pdf

Flores, M. (2018). Evaluación de la conductividad térmica y el tiempo de congelamiento, en bloques congelados de trucha arco iris (Oncorhynchus mykiss), en función al tipo de producto y al sistema de congelamiento. Repositorio digital Universidad Nacional de San Agustín. Perú.

Gallardo, D., \& Domínguez, L. (2015). Aplicación de Modelos de Balance de Masa Nutricional para la estimación de descargas en el cultivo de Trucha Arcoíris en ríos de altura del Austro ecuatoriano. Revista Científica $y$ Tecnológica UPSE, 2(3), 1-11. https://doi.org/10.26423/rctu.v2i3.62

Gomez, Y. (2017). Crecimiento de trucha arco iris (Oncorhynchus mykiss) en jaulas flotantes en la etapa de engorde alimentadas ad libitum y convencionalmente, en Chucasuyo-Juli. Repositorio digital Universidad Nacional del Altiplano. Peru.

Imaki A. (2003). Manual de manejo y crianza de trucha Arco Iris. Editorial JICA. Quito, Ecuador.

Marcel, H. (1998). Tratado de la piscicultura. 4a ed. Madrid, España: Editorial S.A. MUNDI-PRENSA LIBROS, 153 -156.

Morales, G. A. (2004). Crecimiento y eficiencia alimentaria de trucha arco iris (Oncorhynchus mykiss) en jaulas bajo diferentes regímenes de alimentación. Repositorio digital Universidad de Buenos Aires. Argentina.

Morales, G. E. (2019). Influencia de la temperatura del agua sobre el comportamiento biológico de la trucha arco iris (Oncorhynchus mykiss) producida 
en Atillo GAD-Guamote. Repositorio digital Escuela Superior Politécnica de Chimborazo. Ecuador.

Zambrano, A. (2013). Proyecto de factibilidad para la producción y comercialización de trucha arco iris en la parroquia de Papallacta, cantón quijos, provincia del napo. Repositorio digital. Universidad Politécnica Salesiana. Ecuador.

Zapata, A. (2015). Respuesta biológica de la trucha arcoíris frente a tres sistemas de alimentación (balanceado, lombrices de tierra y mixto. Repositorio digital. Escuela Superior Politécnica de Chimborazo. Ecuador. 